\title{
PROBLEMS OF THE STARTING AND OPERATING OF HYDRAULIC COMPONENTS AND SYSTEMS IN LOW AMBIENT TEMPERATURE (PART V)
}

\author{
METHODS ENSURING CORRECT START-UP OF HYDRAULIC COMPONENTS OF SHIP'S ONBOARD DEVICES \\ IN LOW AMBIENT TEMPERATURES
}

Ryszard Jasiński

Gdańsk University of Technology, Poland

\begin{abstract}
A large number of hydraulic devices and systems are started in low ambient temperatures. A good example of such a device is the hoisting winch on the ship. Starting hydraulic drive units in thermal shock conditions (rapid supply of hot oil to the cold unit) may lead to incorrect operation of the actuating system, for instance, due to the loss of clearance between cooperating elements. The article presents methods to prevent the disappearance of effective clearance in a hydraulic motor started in thermal shock conditions. For this reason, the structure of the hydraulic satellite motor was complemented by elements creating special channels through which hot oil could flow and additionally heat fixed parts of the motor. This solution ensures faster heating of motor housing, thus decreasing the temperature difference between the housing and the satellites during motor start-up in thermal shock conditions.
\end{abstract}

Keywords: hydraulic drive, hydraulic components, thermal shock, computer simulation

\section{INTRODUCTION}

Hydraulic drive is becoming more and more frequently used for driving ship's onboard devices $[1,2,15]$, including various types of anchor, mooring, trawl-net, and loading winches, as well as deck cranes. The power of the hydraulic unit can reach $500 \mathrm{~kW}$ and more. High efficiency of hydraulic motors makes them competitive with other driving systems.

Compared to the electric drive, the hydraulic drive used in anchor and loading installations reveals the following advantages:

- high energy efficiency from mass or volume unit,

- simple structure,

- simple and effective overload protection,

- possibility to assemble the device from standard components,

- shorter start-up and shut-down times of hydraulic motors, compared to electric motors,

- lower power consumption during start-up.
Basic components of the hydraulic system of the ship's onboard equipment are pumps, hydraulic motors, and valves. In each of those components energy losses take place, which are defined as volumetric, pressure and mechanical losses. Exact values of these losses for a given hydraulic device are determined in steady-state conditions, neglecting, as a rule, the effect of heat transfer inside the device on the total energy loss balance [13, 14, 16-22, 25]. During the hydraulic machine start-up, the heat transfer between the working medium and machine elements affects significantly the efficiency of energy conversion in this machine, especially in the case of cold machine supplied with hot working medium, which was proved in [3-10], among other sources. Therefore, analysing the issues concerning start-ups of hydraulic components of ships onboard devices in low ambient temperatures is advisable and justified.

Some disadvantages of deck installations with hydraulic drives can also be named. One of them is difficult start-up 
in low ambient temperatures. In lower temperatures the resistance of the flow of hydraulic medium through hydraulic installations and components is higher, due to higher viscosity of the medium. Consequently, situations frequently occur in which the heated working medium is supplied to cold onboard units (thermal shock conditions) [3-10,24]. These situations may lead to incorrect operation of the installation, which is impermissible from the safety point of view. Hydraulic motors should meet the requirements of Polish Standard [30] and Polish Register of Shipping [26-28].

The article presents methods to prevent incorrect operation of hydraulic components started in thermal shock conditions.

\section{STARTING HYDRAULIC MOTORS IN THERMAL SHOCK CONDITIONS}

When a hydraulic unit is started in low ambient temperatures, certain difficulties in its correct operation can be observed. Among other cases, Fig. 1, the cold hydraulic motor can be supplied with oil having higher temperature than the motor, all this leading to thermal shock conditions. Moreover, this start-up can be accompanied by uneven heating of component elements, due to different structural and material properties, which is also a possible source of incorrect component operation.

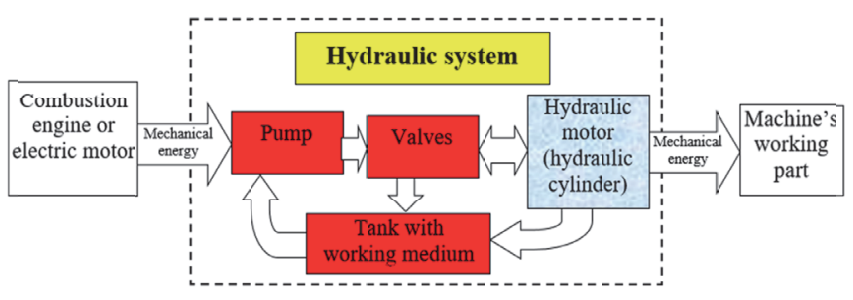

Fig. 1. Structure of hydraulic system: marked cold hydraulic motor supplied with hot working medium [3]

Low-speed hydraulic motors without axial clearance compensation, such as: satellite motor SOK $[29,31]$ or orbital motors [32], can work incorrectly when started in thermal shock conditions. The behaviour of some motors in those conditions and the accompanying phenomena are described in $[3,6-10]$.

\section{METHODS TO PREVENT THE DISAPPEARANCE OF EFFECTIVE CLEARANCE IN HYDRAULIC MOTORS STARTED IN THERMAL SHOCK CONDITIONS}

There are several methods to prevent the disappearance of effective clearance between cooperating elements in hydraulic motors during their start-up in thermal shock conditions. This can be obtained by:

- using a hydraulic motor with a large heat transfer surface between oil and fixed motor elements (housing), which will ensure relatively small changes of effective clearance between cooperating elements during motor start-up,

- designing and manufacturing special motor,

- using structural materials with different linear thermal expansion for hydraulic motor elements,

- additional heating of fixed hydraulic motor elements (housing) before and during the start-up. This can be obtained by using additional elements to create special channels and thus increase the heat transfer surface between the flowing oil and the fixed motor elements (housing),

- electric heating of the housing,

- heating the motor elements before the start-up by hot oil flowing through the inner space of the motor housing (casing space).

\section{MODELS OF HEATING OF HYDRAULIC MOTOR ELEMENTS}

The hydraulic motor comprises two groups of elements: mobile and fixed. Figure 2 shows models of hydraulic motor heating. The difference between them is that in the second model, Fig. 2.B, the fixed elements of the motor (housing) have additional channels through which the hydraulic fluid flows. During the motor start-up in thermal shock conditions,

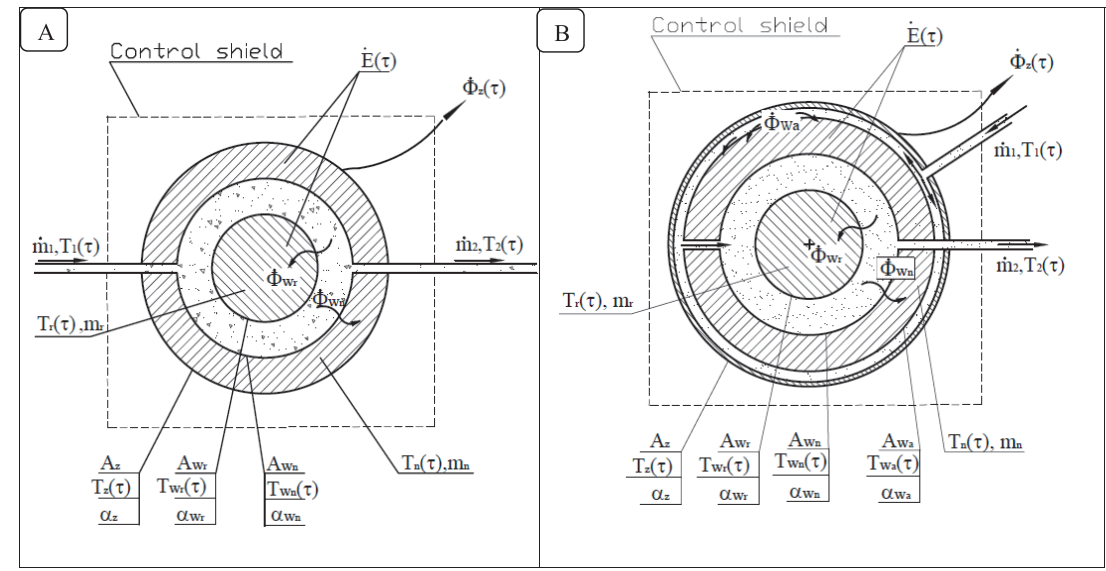

Fig. 2. Models of cold motor heating by the flowing hot fluid $[3,4,6,10]$ :

(A) without additional channels in fixed elements (housing), (B) with additional channels in fixed elements (housing) (A - surface, $T$-temperature, $m$ - mass, $\alpha$ - heat transfer coefficient, $\dot{m}$ - mass flux, $\dot{\Phi}$ - thermal energy flux, $\dot{E}$ flux of energy increase of motor elements,

Symbols: Wn - inner surface of fixed elements without additional channels, Wa-inner surface of additional channels, Wr-outer surface of mobile elements, $z$-outer, $r$ - mobile elements, $n$ - fixed elements, a-additional element (overlay) creating additional channels in fixed elements, 1 - inlet, 2 - outlet 
the hot oil flowing through these channels created by the element placed on the motor intensifies the heating of motor housing. The additional heat transfer surface between the oil and the fixed elements, Fig. 2.B, causes their faster heating than in the case shown in Fig. 2.A.

In the motor model shown in Fig. 2, the inlet and outlet mass fluxes are equal: $\dot{m}_{1}=\dot{m}_{2}$

During the motor start-up in thermal shock conditions, the thermal energy flux from oil $\dot{\Phi}_{\alpha}$ to mobile elements $\dot{\Phi}_{W r}$ and fixed elements $\dot{\Phi}_{W n}$ of the motor without additional channels in housing is equal to $[3,5,6,10,23]$ :

$$
\dot{\Phi}_{\alpha}(\tau)=\dot{\Phi}_{W r}(\tau)+\dot{\Phi}_{W n}(\tau)
$$

$\dot{\Phi}_{\alpha}(\tau)=\alpha_{W r} \cdot A_{W r} \cdot\left(T_{o l}-T_{W r}(\tau)\right)+\alpha_{W n} \cdot A_{W n} \cdot\left(T_{o l}-T_{W n}(\tau)\right)$

where:

$\mathrm{T}_{\mathrm{ol}}$ - average temperature of oil,

r- refers to mobile elements,

n- refers to fixed elements.

The thermal energy flux from oil to fixed elements of the motor with additional channels in housing, $\dot{\Phi}_{N}$, is equal to the sum of the thermal energy flux from oil to the inner surfaces of fixed elements of the motor, $\dot{\Phi}_{W n}$, and that to the surfaces of additional channels, $\dot{\Phi}_{W a}[3,6]$ :

$$
\dot{\Phi}_{N}(\tau)=\dot{\Phi}_{W n}(\tau)+\dot{\Phi}_{W a}(\tau)
$$

Assuming that the conditions of heat transfer from oil to the surfaces of additional channels are similar to those in the channels of fixed motor elements:

$$
\alpha_{W n}=\alpha_{W a} .
$$

Equation (3) can be written in the form:

$$
\dot{\Phi}_{W n}(\tau)+\dot{\Phi}_{W a}(\tau)=\alpha_{W n} \cdot\left(A_{W n}+A_{W a}\right) \cdot\left(T_{o l}-T_{W n}(\tau)\right)
$$

where:

$\mathrm{A}_{\mathrm{Wa}}$ - outer surface of the motor washed by the flowing oil (motor with overlay)

The energy flowing from oil to fixed elements of the motor increases their internal energy (temperature) and the thermal energy flowing out through the outer surface to the environment:

$$
\begin{aligned}
\left(\dot{\Phi}_{W n}(\tau)+\dot{\Phi}_{W a}(\tau)\right) d \tau & =\left(\alpha_{W n} \cdot\left(A_{W n}+A_{W a}\right) \cdot\left(T_{o l}-T_{W n}(\tau)\right)\right) \cdot d \tau= \\
& =m_{n} \cdot c_{n} \cdot d T_{n}+\dot{\Phi}_{Z} \cdot d \tau
\end{aligned}
$$

where:

$c_{n}$ - average specific heat of fixed elements,

$\mathrm{dT}_{\mathrm{n}}$ - increase of average temperature in fixed elements.

In Equation (6), the flux of energy flowing to the environment, $\dot{\Phi}_{z}$, is omitted as very small during the motor start-up, since the external temperature of the motor is approximately equal to the ambient temperature $T_{z}(\tau) \approx T_{o t} \rightarrow \Phi_{z} \approx 0$. The use of overlay decreases the outer surface of the motor which is in direct contact with the environment.

After transforming Equation (6), the relationship between the temperature of fixed elements and time takes the form $[5,10]$

$$
T_{n}(\tau)=T_{o l}-\Delta T_{o l-o t} \cdot e^{-\sigma_{n} \cdot \tau}
$$

where:

$\sigma_{n}=\frac{\alpha_{n} \cdot\left(A_{W n}+A_{W a}\right) \cdot \psi_{n}}{m_{n} \cdot c_{n}}$ - the rate of heating of fixed elements $[3,5,10]$ (the mass of overlay is neglected due to its small contact with fixed elements of the motor),

$\Delta T_{o l-o t}=T_{o l}-T_{o t}$ - difference between oil temperature $\mathrm{T}_{\mathrm{ol}}$ and ambient temperature $\mathrm{T}_{\mathrm{ot}}$ '

$\Psi_{n}, \Psi_{r}-$ coefficient of non-uniform temperature distribution in fixed and mobile motor elements, respectively $[3,5,10]$, $c_{n}, c_{r}$-specific heat of fixed and mobile motor elements, respectively.

The effective clearance $l_{e}$ between cooperating motor elements depends on the assembly clearance $l_{m}$, the difference of thermal expansion $\Delta \mathrm{l}_{t}$ of elements, and deformations of elements $\Delta \mathrm{l}_{\mathrm{p}}$ caused by the action of pressure:

$$
l_{e}(\tau)=l_{m}+\Delta_{p}(\tau)-\Delta_{t}(\tau)
$$

$$
\begin{aligned}
l_{e}(\tau)= & l_{m}+\Delta \nu_{p}(\tau)+\left(T_{o l}-T_{0}\right) \cdot\left(h_{n} \cdot \beta_{n}-h_{r} \cdot \beta_{r}\right)+ \\
& +\Delta T_{o l-o t} \cdot\left(h_{r} \cdot \beta_{r} \cdot e^{-\sigma_{r} \cdot \tau}-h_{n} \cdot \beta_{n} \cdot e^{-\sigma_{n} \cdot \tau}\right)
\end{aligned}
$$

Making use of Equations (7) and (8) along with Equation: $T_{r}(\tau)=T_{o l}-\Delta T_{o l-o t} \cdot e^{-\sigma_{r} \cdot \tau}$, the effective clearance can be given as:

where:

$\sigma_{r}=\frac{\alpha_{r} \cdot A_{W r} \cdot \psi_{r}}{m_{r} \cdot c_{r}}-$ rate of heating of mobile element $[3,5,10]$. 
$\beta_{r}, \beta_{n}$ - linear thermal expansion coefficient of mobile element (r) and fixed element (n), respectively.

When starting the motor in thermal shock conditions, the effective clearance will not disappear $\left(l_{e}>0\right)$, assuming that the effect of pressure on motor elements is small, if the following inequality is fulfilled $[3,10]$ :

$$
\begin{aligned}
& l_{m}+\left(T_{o l}-T_{0}\right) \cdot\left(h_{n} \cdot \beta_{n}-h_{r} \cdot \beta_{r}\right)+ \\
& \quad+\Delta T_{o l-o t} \cdot\left(h_{r} \cdot \beta_{r} \cdot e^{\frac{-\alpha_{r} \cdot A_{W_{r}} \cdot \psi_{r} \cdot \tau}{m_{r} \cdot c_{r}}}-h_{n} \cdot \beta_{n} \cdot e^{\frac{-\alpha_{n} \cdot\left(A_{W_{n}}+A_{W_{a}}\right) \cdot \psi_{n} \cdot \tau}{m_{n} \cdot c_{n}}}\right)>0
\end{aligned}
$$

According to Equation (10), the dimension of the effective clearance is affected by: heat transfer surfaces, masses of elements, specific heats of elements, heat transfer coefficient from oil to motor surfaces, initial clearance, and the difference between oil temperature and ambient temperature.

\section{ADDITIONAL HEATING OF HYDRAULIC MOTOR HOUSING}

The housing of the hydraulic component started in thermal shock conditions can be additionally heated to prevent the disappearance of the effective clearance. This heating can be executed by the flow of hot oil through the inner space of the housing, or heating the housing in another way, electrically, for instance.

Both methods consist in delivering additional heat to the housing (fixed elements) of the hydraulic motor.

The housing of the satellite motor SOK does not have an inner chamber through which hot oil could flow and heat it before the start-up. This solution would allow to increase the temperature of motor elements before the motor start-up in low ambient temperatures. In terms of safety, the best method is the use of an additional element (overlay) placed on the satellite motor SOK to create special channels through which hot oil can flow and heat the outer surface of the motor housing, (Fig. 3). This way the total surface of heat transfer from oil to fixed motor elements (housing) is increased.

Compared do other types of low-speed motor structures, the advantage of satellite motors is their small overall dimension with respect to absorbency, and easy assembling of the overlay over the motor. The article presents a selected structure of the overlay $[4,6,11,12,24]$.

Hot oil flows in through the overlay connector (Fig. 3) and flows around the outer surface of the motor, heating it. After leaving the overlay through the other connector, the oil flows via a pipe (not shown in the figure) to the motor collector. By using the overlay on the satellite motor SOK 100 , the heat transfer surface from oil to fixed elements is increased by nearly twice.

\section{TESTING THE SATELLITE MOTOR SOK IN THERMAL SHOCK CONDITIONS}

Some publications [3-10] describe the performance of the motor SOK 100 (geometric working volume $0,4 \mathrm{dm}^{3} / \mathrm{rev}$ ) in thermal shock conditions, along with the accompanying phenomena. Satellite motors SOK are produced without axial clearance compensation.

The satellite motors SOK are sensitive to thermal shock conditions. The start-up at large temperature difference between hot oil and cold motor can lead to incorrect operation of the motor.

The operation of the satellite motor SOK 100 was tested at the ambient temperature equal to $-20^{\circ} \mathrm{C}$ (in the cooling chamber) and the oil temperature equal to $50^{\circ} \mathrm{C}$.

During this test, incorrect operation of the hydraulic motor was recorded in the form of the disappearance of effective clearances between satellites and covers, and between rotor and covers.

Initially, the pressure at motor inlet increased to about 7,2 MPa (Fig. 4). Then, after about 150 seconds from the start-up, the operation of the motor stabilised. The inlet and outlet pressures were equal to $2 \mathrm{MPa}$, and $1 \mathrm{MPa}$, respectively.

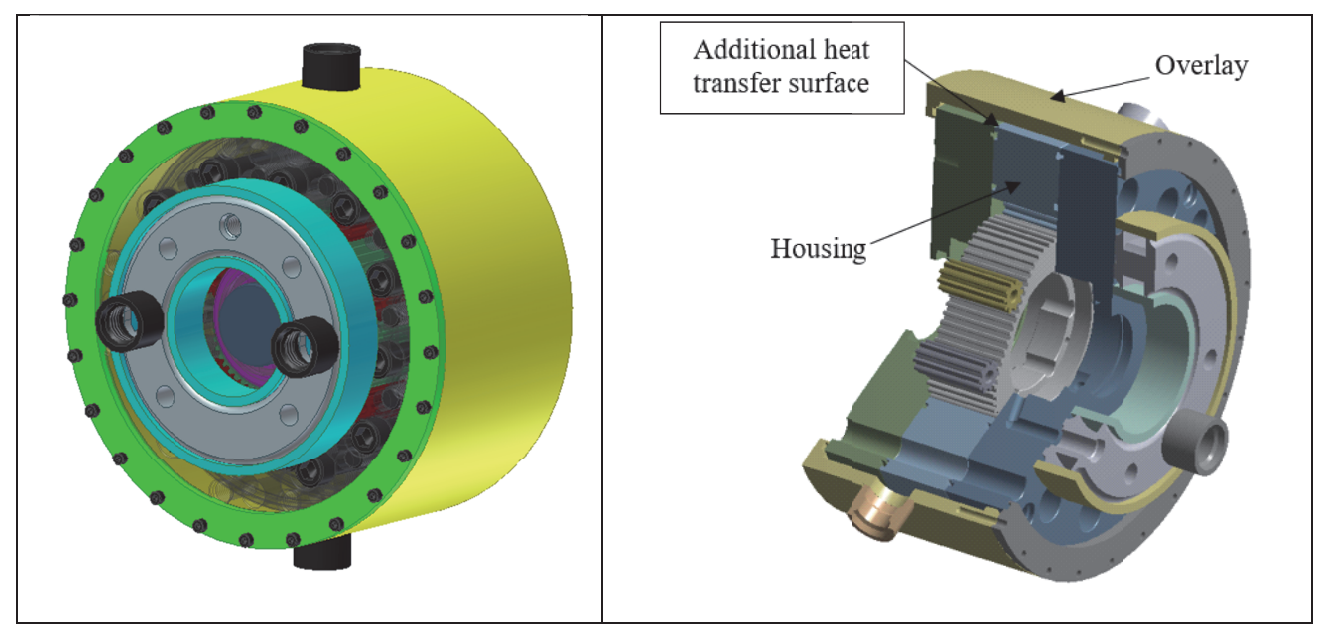

Fig. 3. Model of satellite motor SOK with heating system of fixed elements $[4,6,11,12]$ 


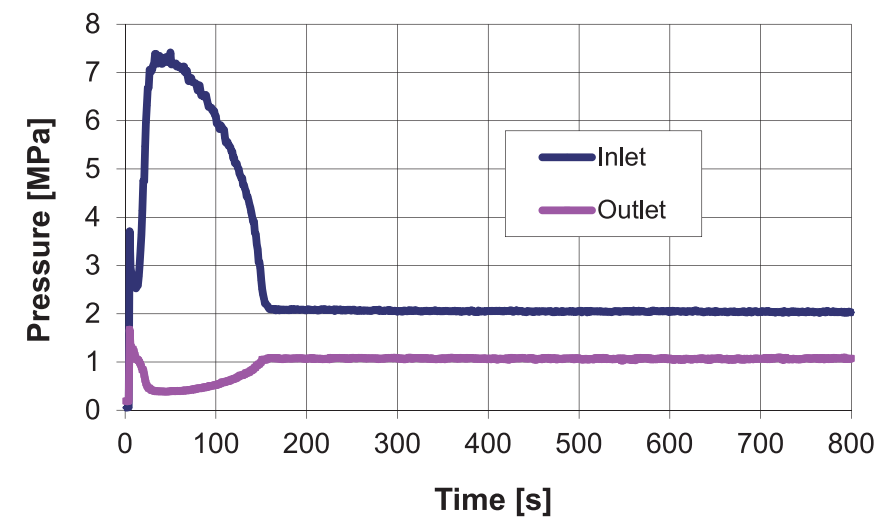

Fig. 4. Inlet and outlet pressure time-histories of unloaded motor [5, 8]

During the first few seconds after the motor start-up, the rotational speed increased to nearly $250 \mathrm{rpm}$ and then decreased to about $90 \mathrm{rpm}$ (Fig. 5). Between second 35 and 150 from the start-up, the rotational speed increased gradually to about $255 \mathrm{rpm}$ and then remained stable.

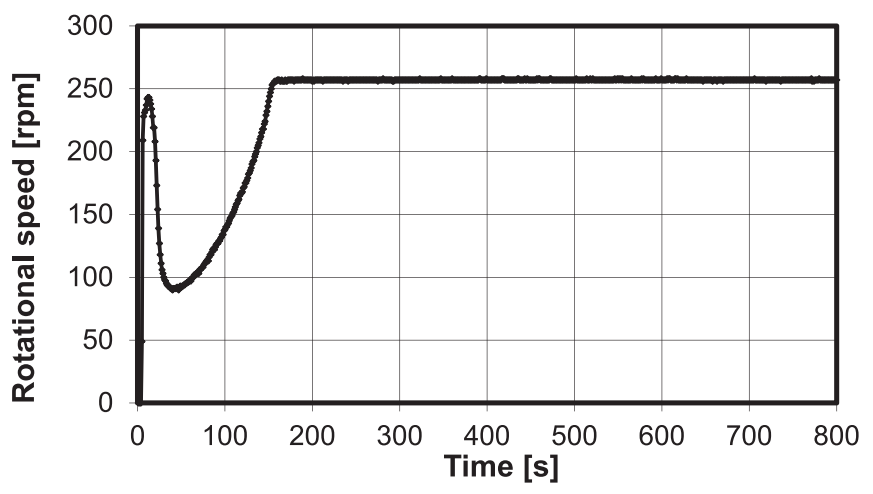

Fig. 5. Time-history of rotational speed [5]

The rotor temperature in the initial time interval increased rapidly to reach $60^{\circ} \mathrm{C}$ after 120 seconds. The friction between the rotor, satellites, and covers led to the temperature increase, mainly in mobile elements. At second 120, the rotor temperature was higher by $10^{\circ} \mathrm{C}$ than the temperature of oil supplied to the motor (Fig. 6).

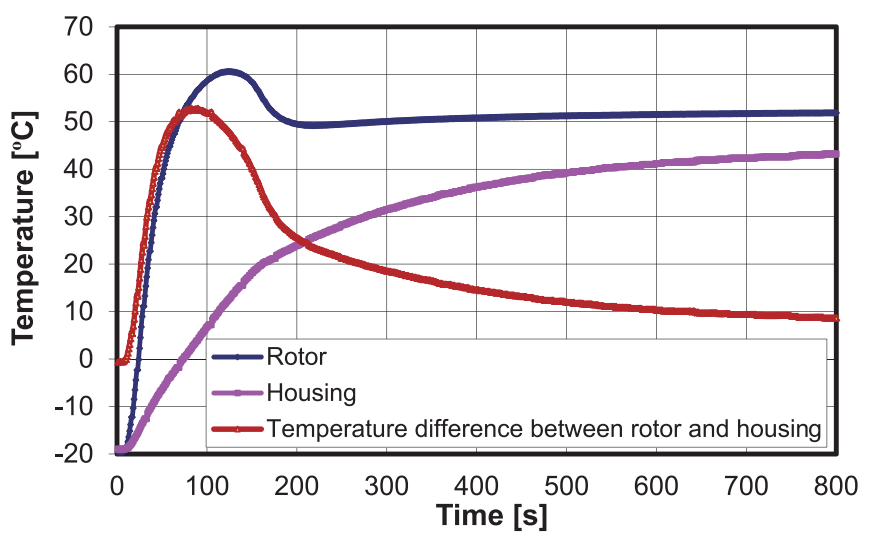

Fig. 6. Temperature of rotor and housing, and temperature difference between these elements, for start-up parameters [5, 9]: initial motor temperature $-20^{\circ} \mathrm{C}$, oil temperature $50^{\circ} \mathrm{C}$, rotational speed in steady-state conditions $255 \mathrm{rpm}$
The effective clearance between the cooperating elements was determined using Equation (8).

At second 60, the effective clearance between the rotor and the covers disappeared, and this state lasted until second 130 (Fig. 7).

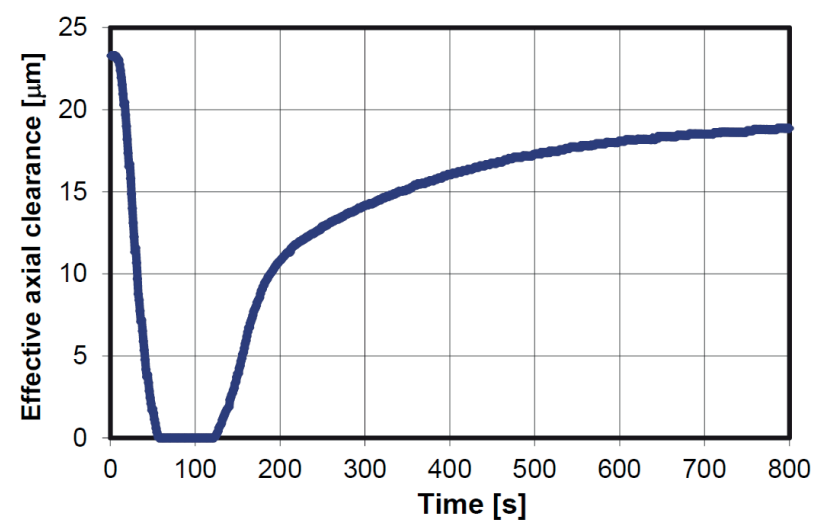

Fig. 7. Effective clearance between rotor and covers, for start-up parameters $[5,9]$ : initial motor temperature $-20^{\circ} \mathrm{C}$, oil temperature $50^{\circ} \mathrm{C}$, rotational speed in steady-state conditions $255 \mathrm{rpm}$

The results of experimental tests made the basis for determining heat transfer coefficients from oil to mobile and fixed elements of the satellite motor SOK 100. These data were used for computer simulation of heating of SOK 100 motor elements.

A computer simulation test was performed for the following start-up parameters: initial motor temperature $-20^{\circ} \mathrm{C}$, oil temperature $50^{\circ} \mathrm{C}$, rotational speed in steady-state conditions $255 \mathrm{rpm}$. The following boundary conditions were assumed: heat transfer coefficient from oil to fixed elements $1460 \mathrm{~W} / \mathrm{m}^{2} \mathrm{~K}$, heat transfer coefficient from oil to mobile elements $1150 \mathrm{~W} / \mathrm{m}^{2} \mathrm{~K}$, and heat transfer coefficient from outer surface to environment $12 \mathrm{~W} / \mathrm{m}^{2} \mathrm{~K}$.

Based on the obtained results of computer simulation, including temperature characteristics of mobile and fixed motor elements, the analysis of the course of motor heating was performed. The element which warmed up the fastest was the satellite. At second 80 , in nominal operating conditions its temperature should become already stable, (Fig. 8), while during incorrect motor operation, mobile elements warmed up to temperatures higher than the oil temperature (Fig. 6).

The satellite, rotor, and housing temperature characteristics obtained from computer simulation (Fig. 8) were used for determining temperature differences between satellites and housing, and between rotor and housing, (Fig. 9). 


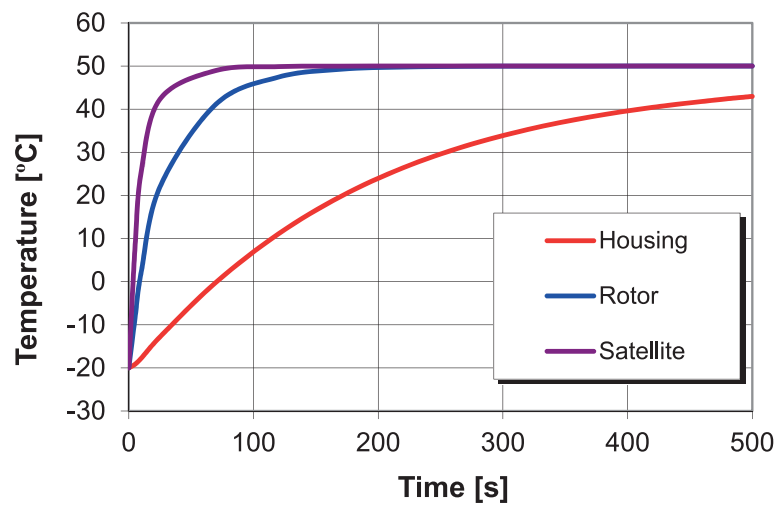

Fig. 8. Temperature changes in housing, rotor and satellite obtained from computer simulation, for start-up parameters: initial motor temperature $-20^{\circ} \mathrm{C}$, oil temperature $50^{\circ} \mathrm{C}$, rotational speed in steady-state conditions $255 \mathrm{rpm}$

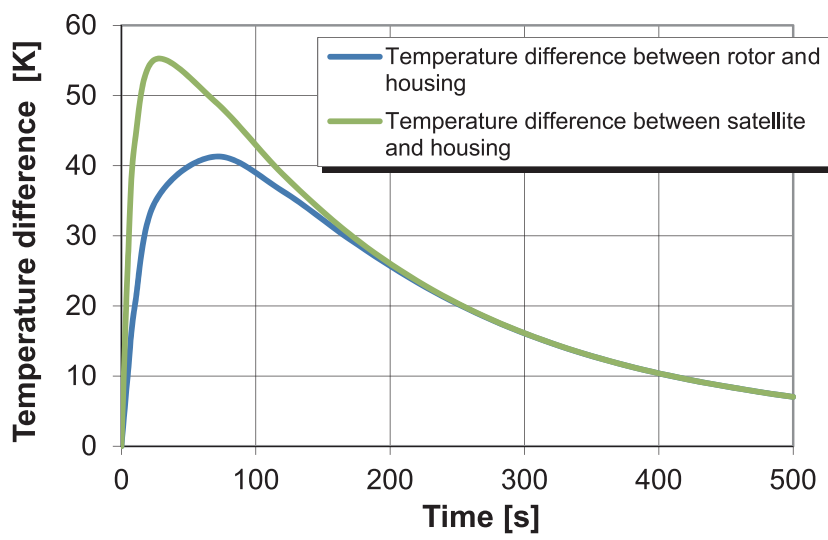

Fig. 9. Temperature differences between rotor and housing, and between satellite and housing, for start-up parameters: initial motor temperature $-20^{\circ} \mathrm{C}$, oil temperature $50^{\circ} \mathrm{C}$, rotational speed in steady-state conditions $255 \mathrm{rpm}$

Based on the temperature difference between satellite and housing, the effective clearance between satellites and covers was calculated from Equation (8). At second 15, the effective clearance between satellites and covers disappeared, and this lack of clearance lasted until second 75 (Fig. 10). In fact, incorrect motor operation could last longer, as the computer simulation neglected the heat of friction between satellites and covers, and between rotor and covers.

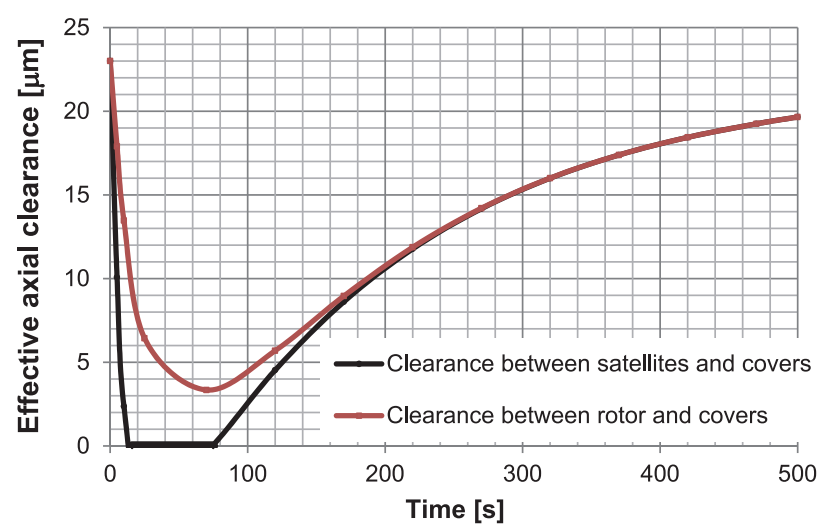

Fig. 10. Effective clearance between satellites and covers, and between rotor and covers, for start-up parameters [6]: initial motor temperature $-20^{\circ} \mathrm{C}$, oil temperature $50^{\circ} \mathrm{C}$, rotational speed in steady-state conditions $255 \mathrm{rpm}$

\section{ANALYSING THE HEATING PROCESS DURING START-UP OF SATELLITE MOTOR SOK WITH ADDITIONAL HEATING OF HOUSING IN THERMAL SHOCK CONDITIONS}

A computer simulation test was performed to study the heating of elements of the satellite motor SOK 100 with additional heating of housing executed with the aid of an overlay. The test was performed for the same start-up conditions in which incorrect motor operation had been recorded: ambient temperature $-20^{\circ} \mathrm{C}$, oil temperature $50^{\circ} \mathrm{C}$, and rotational speed in steady-state conditions $255 \mathrm{rpm}$ (Fig. 11).

The shape of the overlay was designed in such a way as to obtain a similar average flow velocity in the channel created by the overlay and the housing to that observed in inner motor channels. This assumption justified assuming the same value of heat transfer coefficient as for the inner motor channels, i.e. $1460 \mathrm{~W} / \mathrm{m}^{2} \mathrm{~K}$. For mobile elements, the assumed heat transfer coefficient from oil to elements was $1150 \mathrm{~W} / \mathrm{m}^{2} \mathrm{~K}$, while on the outer motor and overlay surfaces this coefficient was assumed equal to $12 \mathrm{~W} / \mathrm{m}^{2} \mathrm{~K}$.

The use of an additional channel in the motor through which hot oil could flow resulted in faster heating of the motor housing. In Fig. 12, after second 320 from motor start-up, steady-state heat flow conditions can be already observed, unlike the case of motor without overlay (Fig. 6).

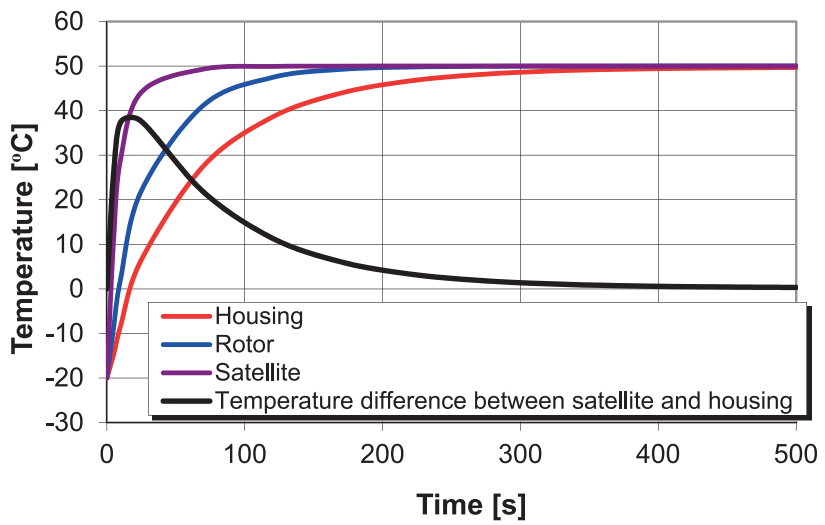

Fig. 12. Temperature changes in housing, rotor and satellite, and temperature difference between satellite and housing during start-up of satellite motor SOK 100 with overlay

The highest temperature difference between satellite and housing was recorded at second 18 and was equal to $38 \mathrm{~K}$ (Fig. 12). For the assumed high difference between the cold motor SOK 100 and the supplying hot oil, which amounted to $70 \mathrm{~K}$, the effective clearance between satellites and covers was not entirely closed. It only decreased by $18 \mu \mathrm{m}$ at second 18 , to the value of $5 \mu \mathrm{m}$ (Fig. 13). 


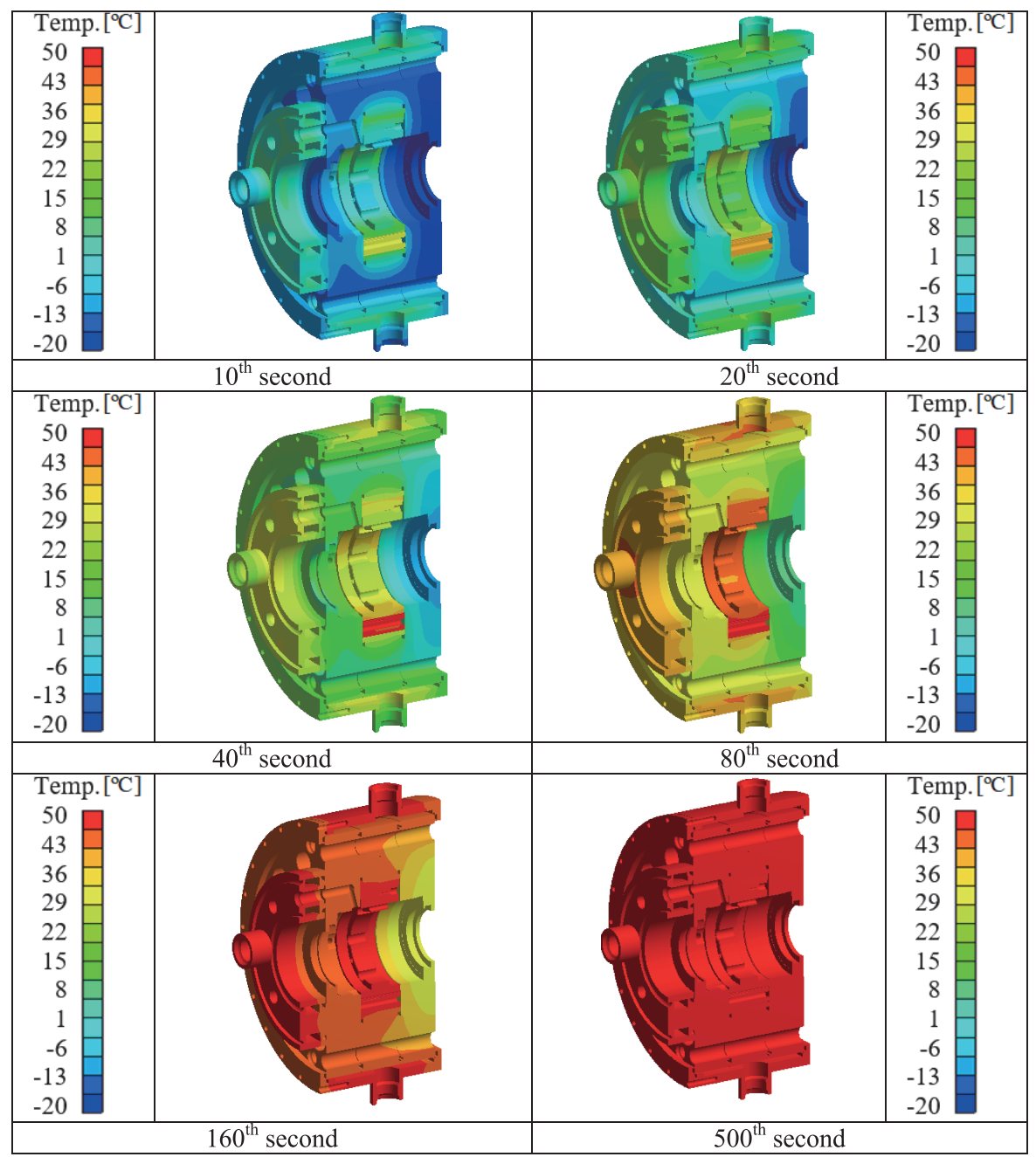

Fig. 11. Temperature distribution in warmed elements of satellite motor SOK 100 with overlay (initial motor temperature $-20^{\circ} \mathrm{C}$, rotational speed in steady-state conditions 255 rpm) at $10^{\text {th }} 20^{\text {th }}, 40^{\text {th }}, 80^{\text {th }}, 160^{\text {th }}$ and $500^{\text {th }}$ second after the supply of oil of temperature $50^{\circ} \mathrm{C}[6]$

\section{ANALYSING THE HEATING PROCESS OF SOK MOTOR WITH OVERLAY FIXED TO HOISTING WINCH FRAME IN THERMAL \\ SHOCK CONDITIONS}

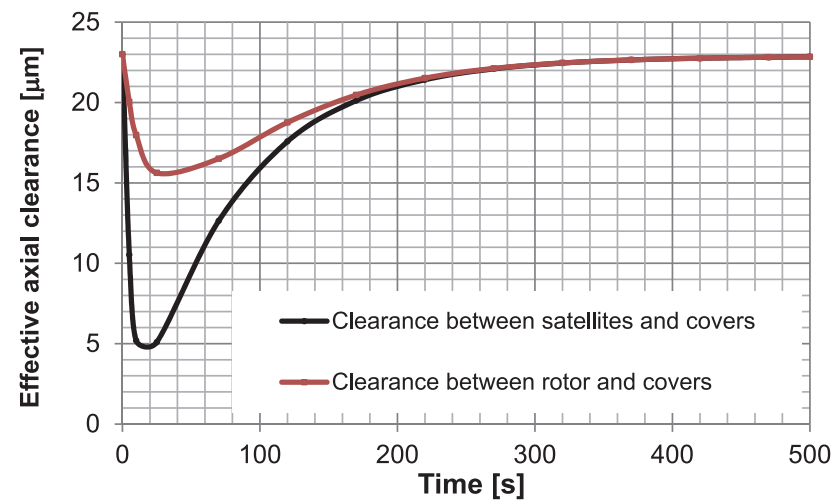

Fig. 13. Effective clearance between satellites and covers, and between rotor and covers, for start-up parameters of motor with overlay [6]: initial motor temperature $-20^{\circ} \mathrm{C}$, oil temperature $50^{\circ} \mathrm{C}$, rotational speed in steady-state conditions $255 \mathrm{rpm}$
Analysing the heating process, in thermal shock conditions, of a motor which is not fixed to any machine or installation frame does not provide comprehensive information on the real course of heating od motor elements, and on changes of effective clearance between cooperating elements. That is why a model test was performed to study heating of elements of the SOK 100 motor with overlay fixed to the hoisting winch frame. During the start-up, the heating of fixed motor elements is expected to take a slower course due to the heat flux flow from these elements to the machine frame.

A computer simulation test was performed for the initial conditions: ambient temperature $-20^{\circ} \mathrm{C}$, oil temperature $50{ }^{\circ} \mathrm{C}$, rotational speed in steady-state conditions $255 \mathrm{rpm}$.

A numerical model of SOK 100 motor fixed to the frame being structural part of the hoisting winch was developed. The following boundary conditions were assumed in the calculations: heat transfer coefficient from oil to fixed elements equal to $1460 \mathrm{~W} / \mathrm{m}^{2} \mathrm{~K}$, heat transfer coefficient from oil to mobile elements equal to $1150 \mathrm{~W} / \mathrm{m}^{2} \mathrm{~K}$, and heat 


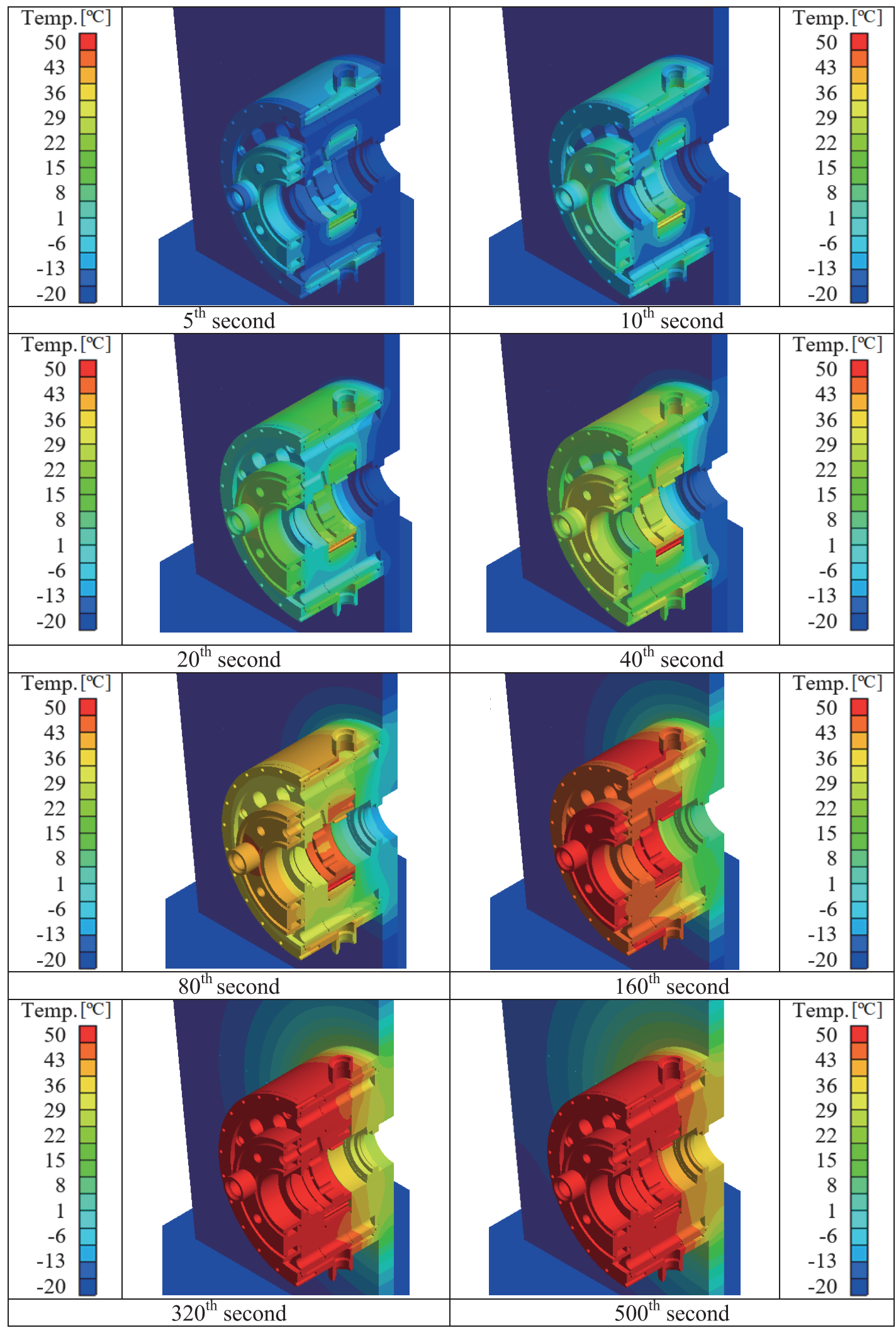

Fig. 14. Temperature distribution in warmed elements of satellite motor SOK 100 with overlay fixed to hoisting winch frame (initial temperature $-20^{\circ} \mathrm{C}$, rotational speed in steady-state conditions $255 \mathrm{rpm}$ ) at $5^{\text {th }} 10^{\text {th }}, 20^{\text {th }}, 40^{\text {th }}, 80^{\text {th }}, 160^{\text {th }}, 320^{\text {th }}$ and $500^{\text {th }}$ second after the supply of oil of temperature $50^{\circ} \mathrm{C}$

transfer coefficient from outer surface to environment equal to $12 \mathrm{~W} / \mathrm{m}^{2} \mathrm{~K}$.

In Fig.14, the flow of heat flux from the motor (front plate) to the hoisting winch frame appears only at second 20 from the start-up and then gradually increases. After 320 seconds, the temperature of some fixed elements of the motor stabilises, while the warming up of the frame is continuing. 
For the same start-up conditions, Fig. 15 shows the housing temperature time-histories for three models of satellite motor SOK 100:

- without overlay and without fixing to the hoisting winch frame,

- with overlay but without fixing to the hoisting winch frame, - with overlay and fixed to the hoisting winch frame.

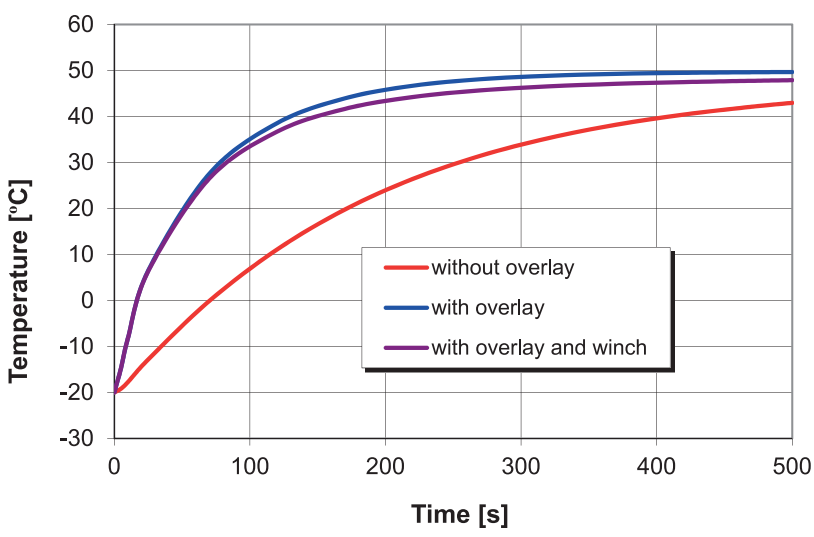

Fig. 15. Temperature of SOK motor housing during start-up in thermal shock conditions, for start-up parameters: initial motor temperature $-20^{\circ} \mathrm{C}$, oil temperature $50^{\circ} \mathrm{C}$, rotational speed in steady-state conditions $255 \mathrm{rpm}$

The heating process of the SOK motor without overlay takes a much slower course that that of the motor with overlay. Additional elements which create channels through which hot oil flows make it possible to affect the operation of hydraulic components in different ambient conditions and the accompanying phenomena.

Fixing the motor to the hoisting winch frame does not cause considerable outflow of heat (and resultant cooling of motor housing), especially at the beginning of the start-up, when incorrect operation of the motor is mostly expected to occur. After 100 seconds, the housing temperature difference for the motor with overlay fixed and not fixed to the hoisting winch frame approximately equalled $2 \mathrm{~K}$. This suggests that neglecting the heat outflow to the hoisting winch frame when modelling the process of motor heating in thermal shock conditions does not cause large error in assessing permissible conditions of correct start-up.

\section{CONCLUSIONS}

The structure of hydraulic motors affects their clearance change limits and, consequently, their operation in low ambient temperatures. When starting the motor in thermal shock conditions, the effective clearance between cooperating elements can totally disappear.

By using additional inner channels in the motor housing, the motor can be protected against the disappearance of clearances between cooperating elements (incorrect motor operation) during the start-up in thermal shock conditions.

Placing an overlay on the hydraulic motor increases the surface of heat transfer from hot oil, which contributes to better protection of the motor against incorrect operation in thermal shock conditions, as shown for the case of the motor SOK 100 as an example.

The use of overlay on the motor increases the oil flow resistance and overall dimensions of the hydraulic component. The increase of oil flow resistance can be large when the motor is started in low ambient temperatures.

The most effective method to start the motor in thermal shock conditions is its earlier heating by the how working medium flowing through the inner space of the housing (casing space) or through additional channels of the motor. Although it extends the time of motor preparation for correct start-up, this method is very effective, as it does not generate additional oil flow resistance during the start-up.

\section{BIBLIOGRAPHY}

1. Dymarski C.: An integrated hydraulic drive system of a novel device for launching lifeboats from large passenger ship. Polish Maritime Research. Vol. 19, 1/2012.

2. Dziewulski W.: Outline of machine hydraulics in shipbuilding industry (in Polish), Wydawnictwo Morskie, Gdansk 1985.

3. Jasiński R.: Influence of design of hydraulic motors on their operation in low ambient temperatures (in Polish). International Scientific-Technical Conference „Hydraulics and Pneumatics'2007", Wroclaw, 2007.

4. Jasiński R.: Research and method for assessment of operation of hydraulic drive components started in low ambient temperature and supplied with hot working medium (in Polish). Report on execution of the research project financed by Polish Ministry of Science and Higher Education (in Polish), No. 4 T07C042 30, Gdansk 2010.

5. Jasiński R.: Operation of selected low-speed hydraulic motors in thermal shock conditions (in Polish). Ph.D. thesis, supervisor: A. Balawender. Gdansk 2002.

6. Jasiński R.: Influence of design of hydraulic components on their operation in low ambient temperatures. Key Engineering Materials, Fundamentals of Machine Design, Vol. 490, 2012.

7. Jasiński R.: Methodology of examination of low-speed hydraulic motors in thermal shock conditions (in Polish), IX Seminar on drives and control 2003, Gdansk, 2003.

8. Jasiński R.: Problems of the starting and operating of hydraulic components and systems in low ambient temperature (Part I). Polish Maritime Research, 4/2008.

9. Jasiński R.: Problems of the starting and operating of hydraulic components and systems in low ambient temperature (Part II). Determining the clearance between 
cooperating elements during the hydraulic components start-up in extremely low ambient temperatures on the grounds of experimental research. Polish Maritime Research, 1/2009.

10. Jasiński R.: Problems of the starting and operating of hydraulic components and systems in low ambient temperature (Part III). Methods of determining parameters for correct start-ups of hydraulic components and systems in low ambient temperatures. Polish Maritime Research, $4 / 2009$.

11. Jasiński R.: System for heating a cold hydraulic satellite motor (in Polish). Patent no. 214740. Patent application no. P.393921 accepted by UP RP in 2011.

12. Jasiński R., Zelewski J.: Protection system for a hydraulic satellite motor started in thermal shock conditions (in Polish). Patent PL 224464. Patent application P393922 accepted by UP RP in 2011.

13. Landvogt B.; Osiecki L.; Patrosz P.; Zawistowski T.; Zylinski B.: Numerical simulation of fluid-structure interaction in the design process for a new axial hydraulic pump. Progress in Computational Fluid Dynamics. Vol. 14, No. 1, 2014. Doi: 10.1504/PCFD.2014.059198.

14. Pobedza J., Sobczyk A.: Properties of high pressure water hydraulic components with modern coatings. Advanced Materials Research. Trans Tech Publications Ltd, 849/2014. doi: 10.4028/www.scientific.net/AMR.849.100.

15. Smotrycki S.: Marine hydraulic drives (in Polish), Wydawnictwo Morskie, Gdansk, 1970.

16. Śliwiński P.: Influence of water and mineral oil on the leaks in satellite motor commutation unit clearances. Polish Maritime Research, vol. 24, 3(95) 2017. Doi: 10.1515/ pomr-2017-0090.

17. Śliwiński P.: The influence of water and mineral oil on the mechanical losses in a hydraulic motor. Chinese Journal of Mechanical Engineering. Article accepted for publication.

18. Śliwiński P.: The influence of water and mineral oil on volumetric losses in a hydraulic motor. Polish Maritime Research, vol. 24, 2017. Doi: 10.1515/pomr-2017-0041.

19. Śliwiński P.: The basics of design and experimental tests of the commutation unit of a hydraulic satellite motor. Archives of Civil and Mechanical Engineering, No 16/2016, DOI: 10.1016/j.acme.2016.04.003.

20. Śliwiński P.: The flow of liquid in flat gaps of satellite motors working mechanism. Polish Maritime Research, No 2/2014.

21. Śliwiński P.: New satellite pumps. Key Engineering
Materials, No 490/2012.

22. Walczak P., Sobczyk A.: Simulation of water hydraulic control system of Francis turbine. American Society of Mechanical Engineers, 2014. doi: dx.doi.org/10.1115/ FPNI2014-7814.

23. Wiśniewski S., Wiśniewski T.: Heat transfer (in Polish). WNT, Warsaw 2000.

24. Zelewski J.: Modernisation project of a satellite motor without axial clearance compensation, resistant to thermal shock conditions, in application to hydraulic ship deck hoisting winch (in Polish). Diploma thesis, supervisor: R. Jasiński, Gdansk University of Technology, 2004.

25. Złoto T., Nagorka A.: An efficient FEM for pressure analysis of oil film in a piston pump. Applied Mathematics and Mechanics, vol.30, No 1/2009.

26. Environmental tests of ship equipment (in Polish). Polish Register of Shipping, Gdansk, 1975.

27. Polish Register of Shipping, "Publication 11P" (in Polish), 1994.

28. Polish Register of Shipping, "Rules for the Classification and Construction of Sea-going Ships" (in Polish), 2002.

29. Technical information documentation: Hydraulic motors SOK produced by ZUO Hydroster (in Polish).

30. PN-86/M-73079 "General conditions of use of unified hydraulics system" (in Polish).

31. Information on http://hydro-precyzja.pl.

32. Catalogues of companies: Bosch-Rexroth, Sauer-Danfoss, Parker.

\section{CONTACT WITH THE AUTHOR}

\author{
Ryszard Jasiński \\ Gdańsk University of Technology \\ 11/12 Narutowicza St. \\ 80 - 233 Gdańsk \\ POLAND
}

